\title{
$S$-CLOSED SPACES
}

\author{
TRAVIS THOMPSON
}

\begin{abstract}
A topological space $X$ is said to be $S$-closed if and only if for every semiopen cover of $X$ there exists a finite subfamily such that the union of their closures cover $X$. For a compact Hausdorff space, the concept of $S$-closed is shown to be equivalent to the concepts of extremally disconnected and projectiveness.
\end{abstract}

A Hausdorff space $X$ is $H$-closed if and only if for every open cover $\left\{U_{a} \mid a \in \Lambda\right\}$ there exists a finite subfamily $\left\{U_{a_{i}} \mid i=1,2, \ldots, n\right\}$ such that the union of their closures cover $X$. In this paper, we expand this concept using semiopen sets.

Definition 1. A set $A$ in a topological space $X$ is semiopen if and only if there exists an open set $V$ such that $V \subset A \subset \bar{V}$, where $\bar{V}$ is the closure of $V$.

Definition 2. A filterbase $F=\left\{A_{a}\right\} s$-converges to a point $x_{0} \in X$ if for each semiopen set $V$ containing $x_{0}$ there exists an $A_{a} \in F$ such that $A_{a} \subset \bar{V}$.

Definition 3. A filterbase $F=\left\{A_{a}\right\} s$-accumulates to a point $x_{0} \in X$ if for each semiopen set $V$ containing $x_{0}$ and $A_{a} \in F, A_{a} \cap \bar{V} \neq \varnothing$.

The corresponding definitions using nets are apparent and will not be stated. An easy consequence of these definitions is

THEOREM 1. Let $F$ be a maximal filterbase in $X$. Then $F s$-accumulates to a point $x_{0} \in X$ if and only if $F$-converges to $x_{0}$.

Definition 4. A topological space $X$ is $S$-closed if and only if for every semiopen cover $\left\{U_{a} \mid a \in \Gamma\right\}$ of $X$ there exists a finite subfamily $\left\{U_{a_{i}} \mid i=1\right.$, $2, \ldots, n\}$ such that the union of their closures cover $X$.

It is apparent from the definition above that a Hausdorff $S$-closed space is $H$-closed. The reader can readily find examples to show that the converse need not be true. Our first result lies in Theorem 2 which characterizes $S$-closed spaces.

THEOREM 2. For a topological space the following are equivalent:

(i) $X$ is $S$-closed.

(ii) For each family of semiclosed sets $\left\{F_{a}\right\}$ (i.e., each $F_{a}$ is the complement of a semiopen set) such that $\cap\left(F_{a}\right)=\varnothing$, there exists a finite subfamily $\left\{F_{a_{i}}\right\}_{i=1}^{n}$ such that $\cap_{i=1}^{n}\left(F_{a_{i}}\right)^{0}=\varnothing$.

Received by the editors December 1, 1975.

AMS (MOS) subject classifications (1970). Primary 54D20, 54D30; Secondary 54G05.

Key words and phrases. $S$-closed, extremally disconnected, projective. 
(iii) Each filterbase $F=\left\{A_{a}\right\}$ s-accumulates to some point $x_{0} \in X$.

(iv) Each maximum filterbase $F$ s-converges.

Proof. (i) $\Rightarrow$ (iv). Let $F=\left\{A_{a}\right\}$ be a maximum filterbase. Suppose that $F$ does not $s$-converge to any point; therefore, by Theorem $1, F$ does not $s$-accumulate to any point. This implies that for every $x \in X$, there exists a semiopen set $V(x)$ containing $x$ and an $A_{a(x)} \in F$ such that $A_{a(x)} \cap \overline{V(x)}=$ $\varnothing$. Obviously $\{V(x) \mid x \in X\}$ is a semiopen cover for $X$ and by hypothesis there exists a finite subfamily such that $\cap_{i=1}^{n} \overline{V\left(x_{i}\right)}=X$. Since $F$ is a filterbase, there exists an $A_{0} \in F$ such that $A_{0} \subset \cap_{i=1}^{n} A_{a\left(x_{i}\right)}$. Hence, $A_{0} \cap \overline{V\left(x_{i}\right)}=\varnothing, 1 \leqslant i \leqslant n$, which implies $A_{0} \cap\left(\cup_{i=1}^{n} \overline{V\left(x_{i}\right)}\right)=A_{0} \cap X=$ $\varnothing$, contradicting the essential fact that $A_{0} \neq \varnothing$.

(iv) $\Rightarrow$ (iii). Each filterbase is contained in a maximal filterbase.

(iii) $\Rightarrow$ (ii). Let $\left\{F_{a}\right\}$ be a collection of semiclosed sets such that $\cap F_{a}=\varnothing$. Suppose that for every finite subfamily, $\cap_{i=1}^{n}\left(F_{a_{i}}\right)^{0} \neq \varnothing$. Therefore $F=$ $\left\{\cap_{i=1}^{n}\left(F_{a_{i}}\right)^{0} \mid n \in Z^{+}, F_{a_{i}} \in\left\{F_{a}\right\}\right\}$ forms a filterbase. By hypothesis, $F$ s-accumulates to some point $x_{0} \in X$. This implies that for every semiopen set $V\left(x_{0}\right)$ containing $x_{0}, F_{a}^{0} \cap \overline{V\left(x_{0}\right)} \neq \varnothing$, for every $a \in \Lambda$. Since $x_{0} \notin \cap F_{a}$ there exists an $a_{0} \in \Lambda$ such that $x_{0} \notin F_{a_{0}}$. Hence, $x_{0}$ is contained in the semiopen set $X-F_{a_{0}}$. Therefore,

$$
\left(F_{a_{0}}\right)^{0} \cap \overline{\left(X-F_{a_{0}}\right)}=\left(F_{a_{0}}\right)^{0} \cap\left(X-\left(F_{a_{0}}\right)^{0}\right)=\varnothing
$$

contradicting the fact that $F s$-accumulates to $x_{0}$.

(ii) $\Rightarrow$ (i). Let $\left\{V_{a}\right\}$ be a semiopen covering of $X$. Then $\cap\left(X-V_{a}\right)=\varnothing$. By hypothesis, there exists a finite subfamily such that $\bigcap_{i=1}^{n}\left(X-V_{a_{i}}\right)^{0}=$ $\bigcap_{i=1}^{n}\left(X-\bar{V}_{a_{i}}\right)=\varnothing$. Therefore, $\cap_{i=1}^{n} \bar{V}_{a_{i}}=X$, and consequently $X$ is $S$ closed.

THeOREM 3. Each $S$-closed, first countable, regular space is finite.

Proof. Let $X$ be an $S$-closed, first countable, regular space. Suppose, if possible, that $X$ is infinite. Since $X$ is compact, it is not discrete. Thus $X$ has an accumulation point $x$. Let $\left\{U_{n} \mid n \in N\right\}$ be a local base at $x$ such that $U_{1}=X, U_{n}$ is open in $X$ and $\overline{U_{n+1}} \subset U_{n}$ for each $n \in N$. Let $\left\{N_{k} \mid k \in N\right\}$ be a family of pairwise disjoint infinite subsets of $N(N=$ the set of positive integers) such that $\cup\left\{N_{k} \mid k \in N\right\}=N$. For each $k \in N$ we set $V_{k}=\{x\} \cup$ $\cup\left\{\bar{U}_{n}-\bar{U}_{n+1} \mid n \in N_{k}\right\}$. Then $\left\{V_{k} \mid k \in N\right\}$ is a semiopen cover of $X$. If $n \in N$, then $\cup\left\{\bar{V}_{k} \mid k \leqslant n\right\} \neq X$. Thus $X$ is not $S$-closed and this is a contradiction.

COROllaRY. Each S-closed metrizable space is finite.

THeorem 4. No regular space containing a P-point is $S$-closed.

Proof. The proof is omitted, because it is similar to the proof of Theorem 
3 , but the index set of cardinality $\kappa_{1}$ is decomposed into $\kappa_{1}$ sets each of cardinality $\kappa_{1}$.

Corollary. $\beta N-N$ is not $S$-closed.

Proof. Assuming the continuum hypothesis, there are $P$-points in $\beta N-N$ (W. Rudin).

Since every compact, countable, regular space is metrizable, we have from the corollary to Theorem 3 the following.

COROLlaRY. Each infinite, $S$-closed, regular space is uncountable.

Lemma. If $Y$ is a regularly closed subset in an $S$-closed space $X$, then $Y$ is $S$-closed.

Proof. The proof is easy and is thus omitted.

THEOREM 5. Each extremally disconnected, compact space is $S$-closed.

Proof. If $X$ is extremally disconnected, then the closure of an open set is an open set. The interior of a semiopen set is dense in it. We consider $\left\{\overline{U_{t}^{0}} \mid t \in T\right\}$ instead of given semiopen cover.

Corollary. $\beta N$ is $S$-closed.

THEOREM 6. If $X$ is a $S$-closed regular space, then $X$ is extremally disconnected.

Proof. Suppose that $X$ is not extremally disconnected. Then there exists a regular open set $O \subset X$ such that $\bar{O}-O$ and $X-\bar{O}$ are nonempty. Let $x \in \bar{O}-O$. Then for every neighborhood $V$ of $x, V \cap O \neq \varnothing$. Therefore, $F=\{(V \cap O)\}$ forms a filterbase in $\bar{O}$. Since $\bar{O}$ is $S$-closed, $F s$-accumulates to some point $x_{0}$ in $\bar{O}$. Quite obviously, the filterbase $F$ also converges to $x$ in the usual sense. We claim that $x_{0} \notin \bar{O}-O$; for if it were, then $x_{0} \in X-O$ and every member of $F$ would have to intersect $X-O$, an impossibility. Thus, $x_{0} \in 0$. There now exists an open set $U$ such that $x_{0} \in U \subset \bar{U} \subset O$ and $x \in X-\bar{U}$. But since $F$ converges to $x$, there must exist a neighborhood of $x$, say $V$, such that $(V \cap O) \subset X-\bar{U}$. This then would imply that $(V \cap O) \cap \bar{U}=\varnothing$, contradicting the fact that $F s$-accumulates to $x_{0}$. Therefore, our assumption that $X$ is not extremally disconnected is false, and the theorem follows.

THEOREM 7. If $X$ is a Hausdorff $S$-closed space, then $X$ is extremally disconnected.

Proof. Although the proof is not identical to that of Theorem 6, it is quite similar and is thus omitted.

Corollary. Let $X$ be a regular compact space. Then $X$ is $S$-closed if and only if $X$ is extremally disconnected. 
COROLlaRY. The one-point compactifications of discrete spaces are not $S$ closed spaces.

COROllaRY. Each $S$-closed, scattered, regular space is finite.

It is well known for a completely regular space $X$ that $X$ is extremally disconnected if and only if $\beta X$ is extremally disconnected [3, p. 96]. Therefore, we have the following corollaries:

COROLlARY. For a completely regular space $X$ the following are equivalent:

(i) $X$ is extremally disconnected.

(ii) $\beta X$ is extremally disconnected.

(iii) $\beta X$ is $S$-closed.

COROLlaRY. For a compact Hausdorff space, the following are equivalent:

(i) $X$ is extremally disconnected.

(ii) $X$ is projective.

(iii) $X$ is $S$-closed.

Proof. The equivalence of (i) and (ii) are well known.

This last useful corollary allows us to see immediately that $\beta N$ is a $S$-closed space, whereas $\beta Q$ and $\beta R$ are not. Additionally, we see that $\beta Q-Q$ is not $S$-closed since it is not compact, and $\beta R-R$ is not $S$-closed because it is not extremally disconnected.

I would like to thank Professor Rastislav Telgarsky for his invaluable suggestions in preparing this manuscript.

\section{REFERENCES}

1. S. Gene Crossley and S. K. Hildebrand, Semi-closed sets and semi-continuity in topological spaces, Texas J. Sci. 22 (1971), 123-126.

2. , Semi-topological properties, Fund. Math. 74 (1972), no. 3, 233-254. MR 46 \# 846.

3. Leonard Gillman and Meyer Jerison, Rings of continuous functions, Van Nostrand, Princeton, N. J., 1960. MR 22 \#6994.

4. Norman Levine, Semi-open sets and semi-continuity in topological spaces, Amer. Math. Monthly 70 (1963), 36-41. MR 29 \#4025.

Department of Mathematics, University of ArKansas, Fayetteville, Arkansas 72701 